\title{
European Scientific Conference on Applied Infectious Disease Epidemiology (ESCAIDE) 2012 - call for abstracts closes 13 July
}

Eurosurveillance editorial team (eurosurveillance@ecdc.europa.eu) ${ }^{1}$

1. European Centre for Disease Prevention and Control (ECDC), Stockholm, Sweden

Citation style for this article:

Eurosurveillance editorial team. European Scientific Conference on Applied Infectious Disease Epidemiology (ESCAIDE) 2012 - call for abstracts closes 13 July. Euro Surveill. 2012;17(28):pii=20218. Available online: http://www.eurosurveillance.org/ViewArticle.aspx?Articleld=20218

Article published on 12 July 2012

Last chance: the call for abstracts for the European Scientific Conference on Applied Infectious Disease Epidemiology (ESCAIDE) closes on Friday 13 July 2012 at 23.00.

For information about ESCAIDE, please consult www. escaide.eu. ECDC also regularly send out information about ESCAIDE via Twitter, on ECDC_EU, \# ESCAIDE 